Article

\title{
Sequential Atmospheric Pressure Plasma-Assisted Laser Ablation of Photovoltaic Cover Glass for Improved Contour Accuracy
}

\section{Christoph Gerhard ${ }^{1,2, *}$, Maximilian Dammann ${ }^{1}$, Stephan Wieneke ${ }^{1,2}$ and Wolfgang Viöl ${ }^{1,2}$}

1 Laboratory of Laser and Plasma Technologies, University of Applied Sciences and Arts, Von-Ossietzky-Straße 99, 37085 Göttingen, Germany;

E-Mails: Maximilian.Dammann@n.hawk-hhg.de (M.D.); wieneke@hawk-hhg.de (S.W.); vioel@hawk-hhg.de (W.V.)

2 Application Center for Plasma and Photonics, Fraunhofer Institute for Surface Engineering and Thin Films, Von-Ossietzky-Straße 100, 37085 Göttingen, Germany

* Author to whom correspondence should be addressed; E-Mail: gerhard@hawk-hhg.de; Tel.: +49-551-370-5376; Fax: +49-551-370-5206.

Received: 28 May 2014; in revised form: 24 June 2014 / Accepted: 26 June 2014 /

Published: 2 July 2014

\begin{abstract}
In this paper, we present sequential atmospheric pressure plasma-assisted laser ablation of photovoltaic cover glass. First, glass samples were plasma pre-treated using a hydrogenous plasma process gas in order to accomplish a modification of the near-surface glass network by a chemical reduction and the implantation of hydrogen. As a result, the transmission at a wavelength of $355 \mathrm{~nm}$ was reduced by approximately $2 \%$ after plasma treatment duration of $60 \mathrm{~min}$. Further, the surface polarity was increased by approximately $78 \%$, indicating an increase of the near-surface index of refraction. Subsequently to the plasma pre-treatment, the samples were laser ablated applying the above-mentioned laser wavelength of a Nd:YAG nanosecond laser. Compared to untreated samples, a significant decrease of the form error by $45 \%$ without any mentionable change in the ablation rate was obtained in the case of pre-treated samples. For comparison, the results and findings are discussed with respect to previous work, where the presented plasma-assisted ablation procedure was applied to optical glasses.
\end{abstract}

Keywords: atmospheric pressure plasma; laser ablation; hybrid ablation; photovoltaic glass; contour accuracy 


\section{Introduction}

In addition to a mechanical protective function against environmental conditions and influences, photovoltaic cover glasses make a considerable contribution to the overall efficiency of photovoltaic cells and assemblies. Against this background, several techniques for improving the illumination of the semiconductor converter such as the use of antireflective (AR) coatings or the direct patterning of cover glass surfaces for light trapping by multiple reflections are in hand [1]. In terms of AR layers, sol-gel coating processes have turned out to be a suitable method for increasing the solar transmittance of cover glasses. Applying this technique, a decrease in reflection by $2.6 \%$ [2,3] to 3\% [4] per surface was already achieved. An increase in transmittance can further be obtained by cover glass surface patterning. Further, a selective guiding of incoming sunlight onto the active photovoltaic converter surfaces can be realized by suitable patterns in order to overcome obscuration effects. Here, prismatic microstructures [5], moth eye surfaces [6] or sub-micrometer diffraction gratings [7,8] were reported to be qualified for photovoltaic applications. Such surface structures can be realized by several manufacturing methods, for example by lithography, milling, compression molding or direct laser structuring. Here, the latter technique represents the most versatile method since no further tools, forms and masks are required. However, precision laser structuring of glasses is a sophisticated task due to the high transparency of optical media. Besides the use of ultraviolet (UV) or infrared (IR) laser irradiation, high-quality laser structuring can be performed by applying ultra-short laser pulses in the picosecond or femtosecond range. Furthermore, hybrid laser ablation methods based on the utilization of absorbing surface-adherent layers or media allow the use of cost-efficient nanosecond laser sources. These methods are known as laser-induced plasma-assisted ablation (LIPAA) [9,10], laser-induced backside wet etching (LIBWE) [11,12], laser etching at a surface adsorbed layer (LESAL) [13,14] and laser-induced backside dry etching (LIBDE) [15]. Recently, nanosecond laser ablation of optical glasses by introducing assisting atmospheric pressure plasmas to the ablation process was investigated. As discussed in the following section, significant improvements were achieved by the use of such assisting plasmas. Against this background, sequential atmospheric pressure plasma-assisted laser ablation of photovoltaic cover glass is reported in the present paper.

\section{Basic Considerations}

In previous work, the influence of hydrogenous cold atmospheric pressure plasmas, generated by dielectric barrier discharge (DBD), on optical and chemical properties of fused silica, which exclusively consists of silicon dioxide $\left(\mathrm{SiO}_{2}\right)$, was investigated. Generally, the gaseous hydrogen $\mathrm{H}_{2}$ from the used process gas $\left(90 \%\right.$ nitrogen $\left.\left[\mathrm{N}_{2}\right], 10 \% \mathrm{H}_{2}\right)$ is dissociated to atomic hydrogen and, as the case may be, partially ionized by such a plasma discharge. As a result, high-reactive hydrogen species are available within the plasma volume and on the fused silica surface, respectively. Two plasma-induced effects were observed by secondary ion mass spectroscopy (SIMS): First, a near-surface chemical reduction of silicon dioxide $\mathrm{SiO}_{2}$ to silicon suboxide $\left(\mathrm{SiO}_{x}\right.$, where $\left.1<x<2\right)$ with an overall thickness of the reduced glass matrix of approximately $100 \mathrm{~nm}$; and second, an implantation of hydrogen into deeper regions of the glass network [16,17]. 
As a result of such plasma-induced modification of the glass network stoichiometry, the optical characteristics were significantly modified. In particular, the UV absorption was temporarily increased, consequently giving rise to an enhanced coupling of incoming UV laser irradiation. This effect was applied in order to develop a novel hybrid laser ablation technique for fused silica, i.e., a combination of hydrogenous atmospheric pressure plasma pre-treatment and subsequent laser ablation. It was shown that in comparison to pure laser ablation, the laser ablation fluence threshold for fused silica could be reduced by a factor of 4.6. Further, the surface roughness of the ablated area was decreased by a factor of $2.3[18,19]$. This ablation technique was also successfully adapted to multi-component optical flint glass where a considerable improvement in contour accuracy (by a factor of 1.9 compared to pure laser ablation) and a mitigation of debris deposition were achieved [20]. On the basis on these promising results the above-described sequential ablation method was applied to photovoltaic cover glass.

\section{Experimental Setup and Procedure}

\subsection{Investigated Glass}

In this work, experiments were performed on 3-mm thick photovoltaic cover glass f|solarfloat from f|solar $\mathrm{GmbH}$. This multi-component glass mainly consists of the network former $\mathrm{SiO}_{2}(72.2 \mathrm{ma} \%)$ and, beyond this, different alkali oxides $\left(\mathrm{M}_{2} \mathrm{O}\right)$ such as sodium oxide $\left(\mathrm{Na}_{2} \mathrm{O}\right)$ or potassium oxide $\left(\mathrm{K}_{2} \mathrm{O}\right)$ as well as divalent oxides $(\mathrm{MO})$, for example calcium oxide $(\mathrm{CaO})$ and magnesium oxide $\mathrm{MgO}$ [21]. As shown in Figure 1, this glass was plasma pre-treated in order to initiate the above-discussed modification of chemical properties (i.e., an implantation of hydrogen into the glass network and the reduction of $\mathrm{SiO}_{2}$ to $\mathrm{SiO}_{x}$ and the accompanying increase in UV-absorption) and to benefit from these modifications during subsequent laser ablation.

\subsection{Plasma Pre-Treatment and Evaluation}

Plasma pre-treatment of $\mathrm{f}$ |solarfloat glass was performed at atmospheric pressure using a conoidal plasma jet source [22], driven at a peak-to-peak voltage $\hat{u}$ of $22 \mathrm{kV}$. The pulse duration $t_{\text {pulse }}$ of each high voltage pulse was $0.8 \mu \mathrm{s}$. As process gas, forming gas 90/10 from Linde (Munich, Germany), i.e., $90 \% \mathrm{~N}_{2}$ (purity 2.8) and $10 \% \mathrm{H}_{2}$, (purity 3.0) at a flow rate of 4 standard litres per minute (slm) was used. The energy per pulse, given by:

$$
E_{\text {pulse }}=\int_{t_{1}}^{t_{2}} u(t) \cdot i(t) \cdot d t=\int_{0 \mu s}^{0.8 \mu s} P(t) \cdot d t
$$

where $u(t)$ is the voltage and $i(t)$ the current of a single plasma pulse, was approximately $100 \mu \mathrm{J}$. Taking the applied plasma pulse repetition rate $f_{\text {rep }}$ of $400 \mathrm{~Hz}$ into account, the averaged plasma power $P_{\text {av }}$ thus amounted to about $40 \mathrm{~mW}$. Since the effective diameter of the plasma jet footprint on the glass sample surfaces, which were placed at a working distance of $1 \mathrm{~mm}$ from the plasma source outlet, was $9 \mathrm{~mm}$, the plasma surface power density $S$ was approx. $63 \mathrm{~mW} \cdot \mathrm{cm}^{-2}$. As shown in previous work, the glass sample surface is not considerably heated by the plasma jet, its maximum temperature $T_{\max }$ amounts to approximately $40{ }^{\circ} \mathrm{C}[23]$. 
In order to determine the impact of such plasma treatment on optical characteristics of the investigated glass, the transmission in the near ultraviolet (UV) and visible (VIS) wavelength range was measured as a function of the plasma treatment duration using an UV/VIS-spectrometer Lambda 650 from Perkin Elmer (Waltham, MA, USA). Further, the total surface energy $\gamma_{\mathrm{s}}$, its polar and disperse fractions, $\gamma_{\mathrm{s}}{ }^{\mathrm{p}}$ and $\gamma_{\mathrm{s}}{ }^{\mathrm{d}}$, and the resulting polarity $P=\gamma_{\mathrm{s}}^{\mathrm{p}} / \gamma_{\mathrm{s}}$ before and after plasma treatment for $60 \mathrm{~s}$ were determined by the Owens-Wendt-Rabel-Kaelble (OWKR) method [24,25]. For this purpose, a Contact Angle Measurement System G10 from Krüss (Hamburg, Germany) was used. In addition, the average area roughness $S_{\mathrm{a}}$ and the root mean square area roughness $S_{\mathrm{q}}$ of an untreated reference sample and after a plasma treatment duration of $60 \mathrm{~min}$ were measured using the built-in atomic force microscope (AFM) of a NeaSNOM microscope from Neaspec GmbH (Munich, Germany).

\subsection{Sequential Laser Ablation and Evaluation}

After plasma pre-treatment for $30 \mathrm{~min}$, ablation experiments were carried out using a 3rd-harmonic Nd:YAG laser Powerlite 9010 from Continuum with a wavelength $\lambda$ of $355 \mathrm{~nm}$ and a pulse duration $\tau$ of 8 ns. As shown in Figure 1, the laser raw beam was focused by a biconvex lens with a focal length of $100 \mathrm{~mm}$, resulting in a focus diameter on the glass sample surface $2 w_{0}$ of $5.8 \mu \mathrm{m}$, where the Rayleigh-length $z_{\mathrm{R}}$ was $0.3 \mathrm{~mm}$. Since the laser source was operated at a fixed output energy $E$ of $9.9 \mathrm{~mJ} \pm 1.2 \mathrm{~mJ}$, the fluence $F$ within the focus was approx. $37.85 \mathrm{~kJ} \cdot \mathrm{cm}^{-2}$. For each ablation spot, single pulses up to a total of 5 pulses were applied.

Figure 1. Principle of sequential atmospheric pressure plasma-assisted laser ablation.

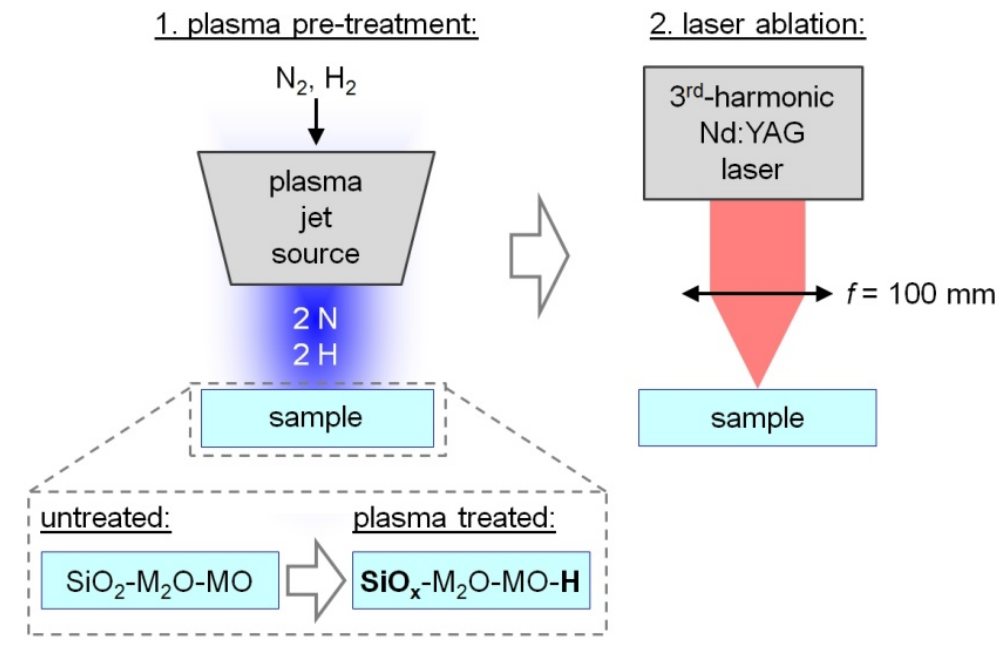

The geometric characteristics of the ablated spots were evaluated using a light microscope Axioskop 2 MAT from Zeiss (Oberkochen, Germany) and a scanning electron microscope (SEM) PSEM eXpress from Aspex (Cheshire, UK). In addition to the ablation spot diameter $\emptyset_{a}$ and the ablation depth $d_{\mathrm{a}}$, the absolute form error $\delta$ was determined according to DIN ISO 1101 . This quality value quantifies the contour accuracy of circular type geometric elements and is given by the difference $\delta=r_{\mathrm{o}}-r_{\mathrm{i}}$ of the radii $r$ of two concentric circles, an outer enveloping circle $\left(r_{\mathrm{o}}\right)$ and an inner minimum circle $\left(r_{\mathrm{i}}\right)$, fitted to the actual irregular spot shape as shown in Figure 2. 
Figure 2. Determination of the absolute form error $\delta$ according to DIN ISO 1101.

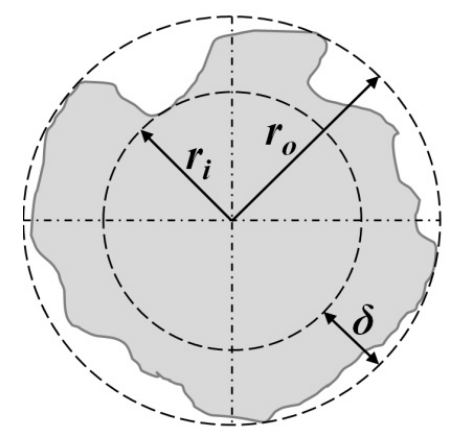

The ablation spot diameter $\varnothing_{\mathrm{a}}$ was defined as the arithmetic mean $\left(2 r_{\mathrm{o}}+2 r_{\mathrm{i}}\right) / 2$ of these radii. Further, the relative form error $\delta_{\text {rel }}=\delta / \emptyset_{\mathrm{a}}$ was calculated.

\section{Results and Discussion}

\subsection{Plasma Pre-Treatment}

By the plasma treatment as described in Section 3.2, two main modifications of the investigated glass were achieved: First, a notable decrease in UV-transmission and second, a significant increase in surface energy and polarity. As shown in figure 3, the maximum change in transmission $\Delta T_{\max }$ of $-2.3 \%$ is found at a wavelength of approximately $330 \mathrm{~nm}$. For technically relevant laser wavelengths, i.e., the third harmonic of Nd:YAG at $355 \mathrm{~nm}$ used in the present work and, for comparison, the xenon-chloride $(\mathrm{XeCl})$ excimer laser wavelength of $308 \mathrm{~nm}$, the decrease in transmission after a plasma treatment duration $t_{\text {plasma }}$ of $60 \mathrm{~min}$ amounts to approximately $2 \%$. The plasma treatment duration-dependent continuous decrease in transmission (Figure 3, right) at those wavelengths can be described by an asymptotic interrelation, theoretically reaching its saturation of $2.14 \%$ after $t_{\text {plasma }} \approx 170 \mathrm{~min}$.

Figure 3. Relative change in transmission $\Delta T v s$. wavelength for three plasma treatment durations (left) and $\Delta T$ at a wavelength of $308 \mathrm{~nm}$ and $355 \mathrm{~nm}$ vs. plasma treatment duration $t_{\text {plasma }}$ (right).
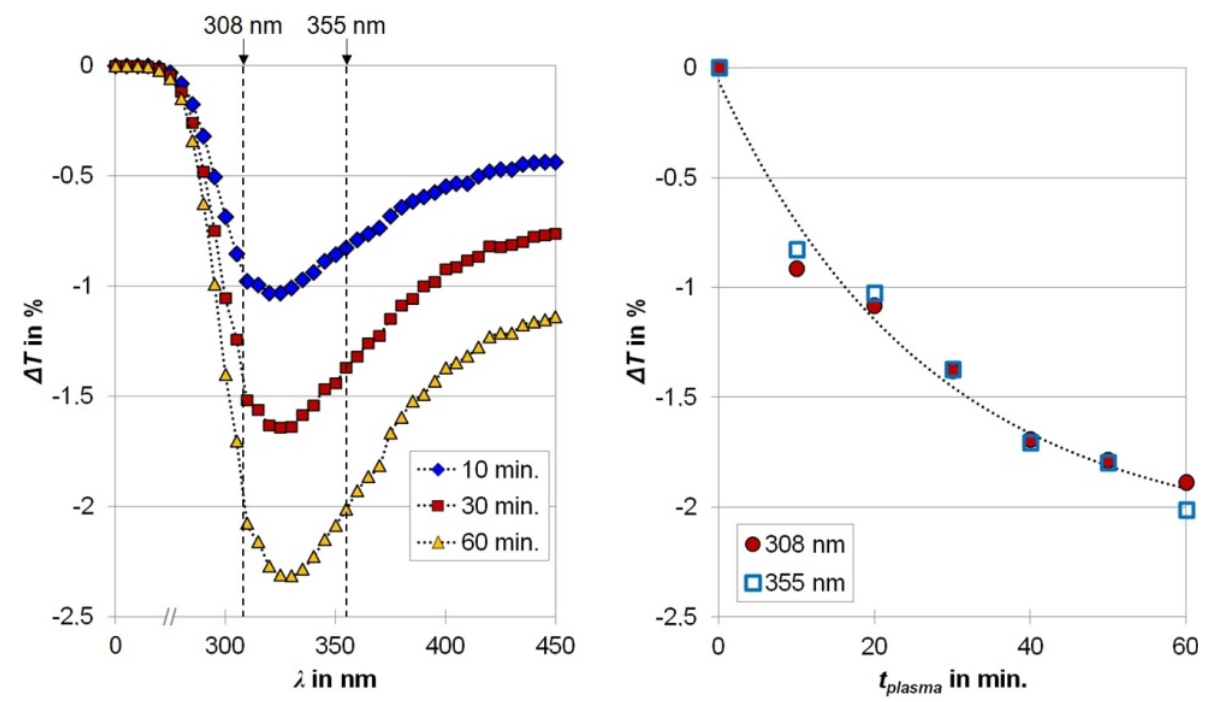
In previous work, a decrease in transmission of $14.1 \%$ was achieved after plasma treatment duration of $15 \mathrm{~min}$ by applying the presented plasma pre-treatment to fused silica, a single-component glass [18]. It can thus be stated that in the present case merely a comparatively low plasma-induced effect on the UV-transmission of the investigated photovoltaic cover glass was obtained. Such behaviour was already observed in the case of another multi-component glass, heavy flint glass SF5 from Schott (Mainz, Germany), where $\Delta T$ amounted to $2.43 \%$ after plasma treatment duration of $30 \mathrm{~min}$ [20]. This could be explained by the fact that the fused silica network provides a considerable number of gaps with a theoretical averaged interatomic distance of $0.163 \mathrm{~nm}$ within the network-forming $\mathrm{SiO}_{4}$ tetrahedrons [26], where hydrogen from the plasma can accumulate quite easily. However, in terms of contour accuracy, laser ablation of SF5 was notably improved in spite of the low plasma-induced decrease in transmission.

The above-mentioned increase in total surface energy of the investigated photovoltaic cover glass is listed in Table 1. It turns out that the polar fraction of the surface energy $\gamma_{\mathrm{s}}^{\mathrm{p}}$ is much more affected by the plasma pre-treatment than the disperse one.

Table 1. Polar $\left(\gamma_{\mathrm{s}}^{\mathrm{p}}\right)$ and disperse $\left(\gamma_{\mathrm{s}}{ }^{\mathrm{d}}\right)$ fraction of surface energy, total surface energy $\gamma_{\mathrm{s}}$ and polarity $P$ of untreated and plasma treated (for $60 \mathrm{~s}$ ) photovoltaic cover glass f|solarfloat including absolute percentage change $\Delta$.

\begin{tabular}{cccc}
\hline Parameter & $\boldsymbol{t}_{\text {plasma }}=\mathbf{0 ~ s}$ & $\boldsymbol{t}_{\text {plasma }}=\mathbf{6 0} \mathbf{s}$ & $\boldsymbol{\Delta}$ in \% \\
\hline$\gamma_{\mathrm{s}}^{\mathrm{p}}$ in $\mathrm{mJ} \cdot \mathrm{m}^{-2}$ & 18.76 & 46.82 & +149.62 \\
$\gamma_{\mathrm{s}}{ }_{\mathrm{s}}$ in $\mathrm{mJ} \cdot \mathrm{m}^{-2}$ & 37.47 & 31.99 & -14.63 \\
$\gamma_{\mathrm{s}}$ in $\mathrm{mJ} \cdot \mathrm{m}^{-2}$ & 56.22 & 78.81 & +40.16 \\
$P=\gamma_{\mathrm{s}}^{\mathrm{p}} / \gamma_{\mathrm{s}}$ & 0.33 & 0.59 & +78.1 \\
\hline
\end{tabular}

This behavior confirms the plasma-induced modification of the optical properties by a modification of the glass network composition and a re-orientation of the involved compounds: Generally, $\gamma_{s}^{p}$ is proportional to the surface polarisability $k_{\mathrm{s}}$ and the molecule polarisability $\alpha$ as suggested by Carré [27]. Further, $\alpha$ is directly related to the index of refraction $n$ according to the Clausius-Mosotti equation, given by:

$$
\frac{n^{2}-1}{n^{2}+2}=\frac{N_{\mathrm{A}} \cdot \rho \cdot \alpha}{3 \cdot \varepsilon_{0} \cdot M}
$$

here, $N_{\mathrm{A}}$ is the Avogadro constant, $\rho$ is the density, $\varepsilon_{0}$ is the vacuum permittivity and $M$ the molar mass [28]. Both observed effects, the decrease in transmission, which partially corresponds to an increase in absorption, and the increase in the polar fraction of the surface energy and the index of refraction, respectively, thus confirm the influence of the applied plasma pre-treatment on the optical properties of the investigated photovoltaic cover glass.

In addition to a modification of the optical properties, the surface morphology of the investigated samples was notably affected by the plasma treatment. Such impact of DBD-plasmas on the roughness of glass surfaces was already reported in previous work. It was shown that, depending on the particular plasma discharge type and the used process gas, both surface smoothing [29] and surface 
wrinkling [17] can occur. In the present case, the average area roughness $S_{\mathrm{a}}$ as well as the root mean square area roughness $S_{\mathrm{q}}$ were reduced as a result of the plasma treatment as shown in Figure 4.

Figure 4. Atomic force microscope (AFM)-images of untreated (left) and plasma-treated (right) photovoltaic cover glass surfaces including the particular average area roughness $S_{\text {a }}$ and root mean square area roughness $S_{\mathrm{q}}$.

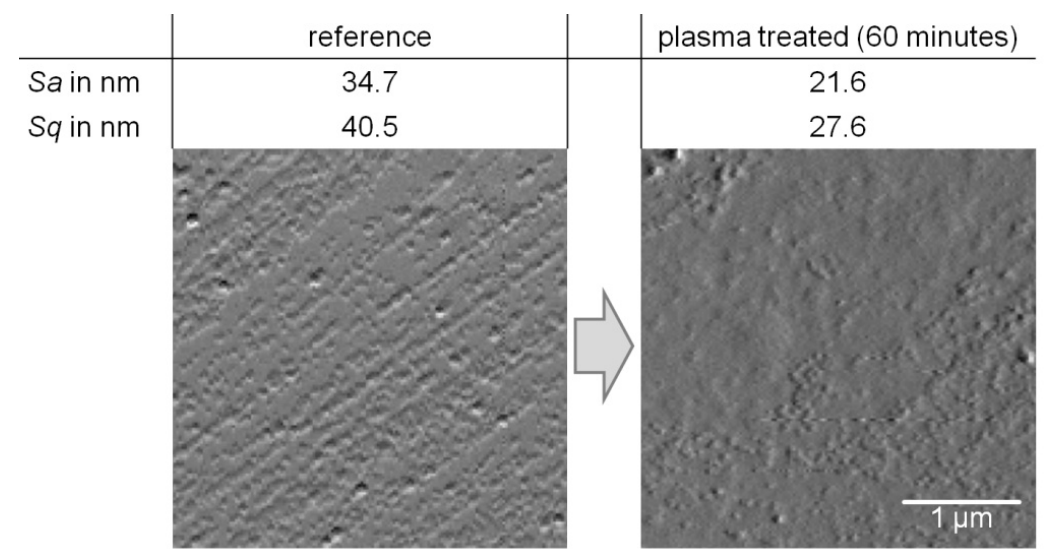

One has to consider that such surface smoothing contributes to the observed increase in surface energy since the surface roughness is directly related to the resulting contact angle of test liquids used for its determination [30]. However, in the case of a pure morphologic surface smoothing, the transmission should rather increase due to a reduction of scattering losses. The above-presented decrease in transmission thus indicates a change in index of refraction as also verified in previous work [16].

\subsection{Sequential Laser Ablation}

The influence of the applied plasma pre-treatment on subsequent laser ablation is summarized by the particular ratios of the investigated parameters in Figure 5. Here, the appropriate values were averaged for 1 to 5 laser pulses and the quotients for plasma-laser ablation and laser ablation (p-1/1) were calculated.

Figure 5. Comparison of laser (1) and sequential plasma-laser (p-1) ablation characteristics by the particular ratio $\mathrm{p}-1 / 1$ for the ablation spot diameter $\varnothing_{\mathrm{a}}$, the ablation depth $d_{\mathrm{a}}$ and the absolute and relative form error, $\delta$ and $\delta_{\text {rel, }}$ averaged for 1 to 5 laser pulses.

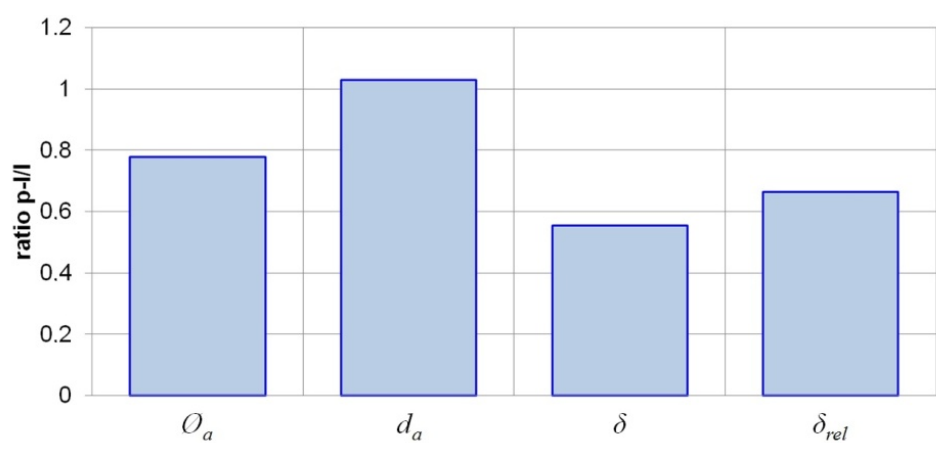

It can be stated that the most significant plasma-induced enhancement concerns the form error values $\delta(-45 \%)$ and $\delta_{\text {rel }}(-34 \%)$, respectively. Beyond, the ablation spot diameter was reduced by 
approximately $22 \%$ whereas the ablation depth was not notably affected $(+3 \%)$ as shown in more detail in Figure 6.

Figure 6. Ablation spot diameter $\varnothing_{\mathrm{a}}$ (left) and ablation depth $d_{\mathrm{a}}$ (right) after laser and sequential plasma-laser ablation $v s$. number of pulses.

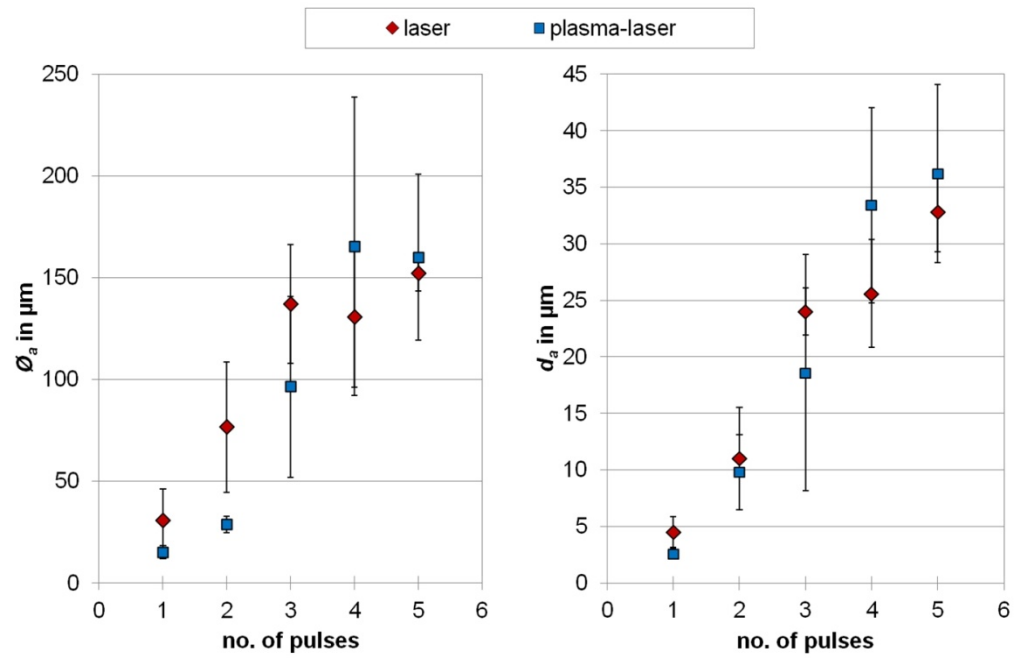

A comparable behavior due to the plasma pre-treatment was already observed in previous work where the impact of sequential plasma-laser ablation on heavy flint glass was investigated. Here, both the ablation spot diameter and depth was marginally influenced, but a significant reduction of the form error by $47 \%$ was achieved [20]. Further, a model test was performed on another photovoltaic cover glass, where the most significant plasma-induced improvement also concerned the form error which was reduced by $58 \%$. The significant reduction in form error is visualized by the comparison of single-pulse laser and sequential plasma-laser ablated spots on f|solarfloat glass surfaces in Figure 7.

Figure 7. Qualitative light (top) and scanning electron microscopic (bottom) comparison of photovoltaic cover glass f|solarfloat surfaces, single-pulse laser ablated (left) and sequential plasma-laser ablated (right) at an energy of $9.9 \mathrm{~mJ} \pm 1.2 \mathrm{~mJ}$.
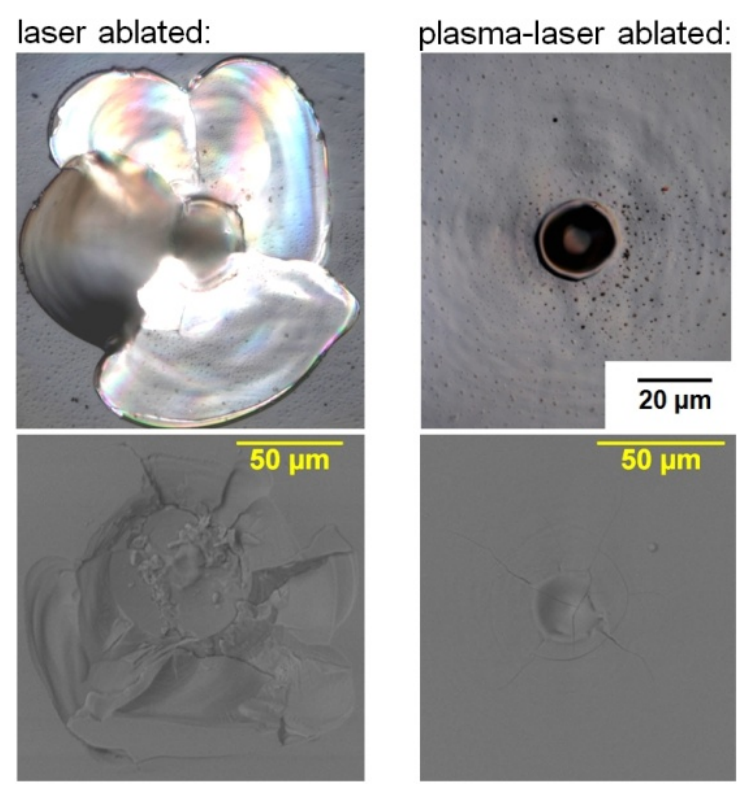
Regarding these microscopic images it turns out that the formation of flaked off glass particles around the ablation spots could be reduced and even avoided by the plasma pre-treatment, most likely due to an improved coupling of incoming laser irradiation into the surface. However, for higher numbers of pulses, such flaking also occurred in the case of sequential plasma-laser ablation. This effect is also quantified by the appropriate values for $\delta$ and $\delta_{\text {rel }}$ in Figure 8 .

Figure 8. Absolute form error $\delta$ (left) and relative form error $\delta_{\text {rel }}$ (right) after laser and sequential plasma-laser ablation $v$ s. number of pulses.
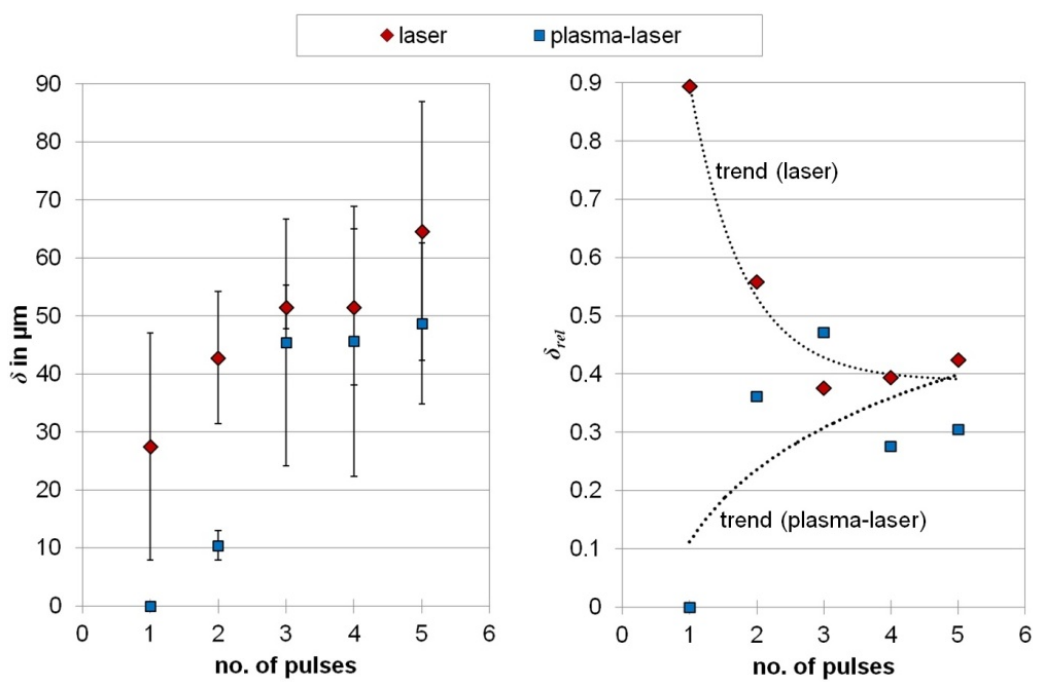

Here, the best results are found for the first two laser pulses, corresponding to a maximum ablation depth of approximately $10 \mu \mathrm{m}$ (compare Figure 6). This is explained by the fact that plasma treatment processes generally provoke near-surface modifications up to a depth of some tens of nanometers as also confirmed by depth-resolved SIMS in previous work (compare Section 2). Thus, the bigger part of the plasma-modified layer, i.e., silicon suboxide and implanted hydrogen, is removed after the first laser pulse. However, it was shown in (previously unreleased) work that the hydrogen implanted by the plasma treatment can act as precursor for a subsequent laser-induced chemical reduction of deeper regions within the glass bulk material [31], giving rise to an improved coupling of laser irradiation. The diffusion depth of hydrogen in glassy materials can amount to several microns. This fact explains the plasma-induced improvements of the contour accuracy up to ablation depth of approximately $10 \mu \mathrm{m}$, which is about 100-times higher than the depth of penetration of the plasma. It can further be assumed that the plasma treatment provokes several secondary effects such as the formation of non-bridging oxygen (NBO) and a modification of the silicon dioxide tetrahedron's bond angles and interatomic distances within a near-surface bulk material layer. Such modifications were already observed in the case of plasma-treated fused silica. For higher ablation depths, where the glass bulk material is not sufficiently influenced by the plasma, the ablation behavior is nearly the same as for untreated glass.

\section{Conclusions}

In the present work it was shown that crucial optical parameters of the investigated photovoltaic cover glass were modified by the applied plasma pre-treatment. In particular, the UV-transmission was 
notably reduced, giving rise to an improved coupling of incoming laser irradiation. For a low number of laser pulses, this effect allows a significant reduction of flaking around the ablation spots and an accompanying decrease in form error without affecting the ablation depth, i.e., the ablation rate.

However, comparatively long-term plasma pre-treatment is required for achieving the observed enhancements of the laser ablation process. In order to improve the efficiency and to shorten the process time of this plasma treatment, further experiments have to be performed. Here, deciding plasma parameters such as the composition of the used process gas, the plasma power and the plasma discharge type shall be modified and optimized.

Another approach for providing an industrial-scale atmospheric pressure plasma-assisted laser ablation method is the application of a simultaneous process instead of a sequential one. The feasibility of such simultaneous plasma-assisted ablation was already shown in previous work where argon was used as process gas [32-34]. One has to consider that in this case, the advantage was taken of plasma-physical mechanisms instead of plasma-chemical effects. The characteristics and performance of a simultaneous process using chemically reactive hydrogenous process gases will be investigated in ongoing work. Such simultaneous process should further be applied in order to improve the ablation quality during multiple-pulse ablation.

\section{Acknowledgments}

This work was supported by the European Regional Development Funds and the Workgroup Innovative Projects of Lower Saxony in the frame of the Lower Saxony Innovation Network for Plasma Technology, project funding reference number W2-80029388. In addition, the authors thank the Volkswagen Foundation and the Federal State Lower Saxony for the support in the frame of the state funding program "Niedersächsisches Vorab". The authors further gratefully acknowledge the support by f|solar $\mathrm{GmbH}$ in Osterweddingen, Germany, by providing photovoltaic cover glass samples and advice.

\section{Author Contributions}

The first draft of the present paper was written by Christoph Gerhard who also carried out the laser ablation experiments together with Maximilian Dammann. The latter further performed the spectroscopic and microscopic measurements, which were then evaluated by both already mentioned authors. The results and findings were finally discussed and interpreted with Stephan Wieneke and Wolfgang Viöl who both supervised the presented work and edited the final version of the manuscript.

\section{Conflicts of Interest}

The authors declare no conflict of interest.

\section{References}

1. Deubener, J.; Helsch, G.; Moiseev, A.; Bornhöft, H. Glasses for solar energy conversion systems. J. Eur. Ceram. Soc. 2009, 29, 1203-1210. 
2. Nostell, P.; Roos, A.; Karlsson, B. Antireflection of glazings for solar energy applications. Sol. Energy Mater. Sol. Cells 1998, 54, 223-233.

3. Nostell, P.; Roos, A.; Karlsson, B. Optical and mechanical properties of sol-gel antireflective films for solar energy applications. Thin Solid Films 1999, 351, 170-175.

4. Gombert, A.; Glaubitt, W.; Rose, K.; Dreibholz, J.; Zanke, C.; Bläsi, B.; Heinzel, A.; Horbelt, W.; Sporn, D.; Döll, W.; Wittwer, V.; Luther, J. Glazing with very high solar transmittance. Sol. Energy 1998, 62, 177-188.

5. Chung, B.C.; Virshup, G.F.; Hikido, S.; Kaminar, N.R. 27.6\% efficiency (1 sun, air mass 1.5) monolithic $\mathrm{Al}_{0.37} \mathrm{Ga}_{0.63} \mathrm{As} / \mathrm{GaAs}$ two-junction cascade solar cell with prismatic cover glass. Appl. Phys. Lett. 1989, 55, 1741-1743.

6. Wilson, S.J.; Hutley, M.C. The optical properties of 'moth eye' antireflection surfaces. Opt. Acta 1982, 29, 993-1009.

7. Gombert, A.; Rose, K.; Heinzel, A.; Horbelt, W.; Zanke, C.; Bläsi, B.; Wittwer, V. Antireflective submicrometer surface-relief gratings for solar applications. Sol. Energy Mater. Sol. Cells 1998, $54,333-342$.

8. Heine, C.; Morf, R.H. Submicrometer gratings for solar energy applications. Appl. Opt. 1995, 34, 2476-2482.

9. Zhang, J.; Sugioka, K.; Midorikawa, K. Direct fabrication of microgratings in fused quartz by laser-induced plasma-assisted ablation with a $\mathrm{KrF}$ excimer laser. Opt. Lett. 1998, 23, 1486-1488.

10. Sugioka, K.; Midorikawa, K. Novel technology for laser precision micro-fabrication of hard materials. RIKEN Rev. 2001, 32, 36-42.

11. Wang, J.; Niino, H.; Yabe, A. One-step microfabrication of fused silica by laser ablation of an organic solution. Appl. Phys. A 1999, 68, 111-113.

12. Ding, X.; Kawaguchi, Y.; Niino, H.; Yabe, A. Laser-induced high-quality etching of fused silica using a novel aqueous medium. Appl. Phys. A 2002, 75, 641-645.

13. Böhme, R.; Zimmer, K. Low roughness laser etching of fused silica using an adsorbed layer. Appl. Surf. Sci. 2004, 239, 109-116.

14. Zimmer, K.; Böhme, R.; Rauschenbach, B. Laser etching of fused silica using an adsorbed toluene layer. Appl. Phys. A 2004, 79, 1883-1885.

15. Hopp, B.; Vass, C.; Smausz, T.; Bor, Z. Production of submicrometre fused silica gratings using laser-induced backside dry etching technique. J. Phys. D 2006, 39, 4843-4847.

16. Gerhard, C.; Tasche, D.; Brückner, S.; Wieneke, S.; Viöl, W. Near-surface modification of optical properties of fused silica by low-temperature hydrogenous atmospheric pressure plasma. Opt. Lett. 2012, 37, 566-568.

17. Gerhard, C.; Weihs, T.; Tasche, D.; Brückner, S.; Wieneke, S.; Viöl, W. Atmospheric pressure plasma treatment of fused silica, related surface and near-surface effects and applications. Plasma Chem. Plasma Process. 2013, 33, 895-905.

18. Brückner, S.; Hoffmeister, J.; Ihlemann, J.; Gerhard, C.; Wieneke, S.; Viöl, W. Hybrid laser-plasma micro-structuring of fused silica based on surface reduction by a low-temperature atmospheric pressure plasma. J. Laser Micro Nanoeng. 2012, 7, 73-76. 
19. Hoffmeister, J.; Gerhard, C.; Brückner, S.; Ihlemann, J.; Wieneke, S.; Viöl, W. Laser micro-structuring of fused silica subsequent to plasma-induced silicon suboxide generation and hydrogen implantation. Phys. Procedia 2012, 39, 613-620.

20. Gerhard, C.; Heine, J.; Brückner, S.; Wieneke, S.; Viöl, W. A hybrid laser-plasma ablation method for improved nanosecond laser machining of heavy flint glass. Lasers Eng. 2013, 24, 391-403.

21. flsolarfloat data sheet; f|solar GmbH: Osterweddingen, Germany, 2013.

22. Brückner, S.; Rösner, S.; Gerhard, C.; Wieneke, S.; Viö1, W. Plasma-based ionisation spectroscopy for material analysis applications. Mater. Test. 2011, 53, 639-642.

23. Gredner, A.; Gerhard, C.; Wieneke, S.; Schmidt, K.; Viöl, W. Increase in generation of poly-crystalline silicon by atmospheric pressure plasma-assisted excimer laser annealing. J. Mater. Sci. Eng. B 2013, 3, 346-351.

24. Kaelble, D.H. Peel adhesion: Influence of surface energies and adhesive rheology. J. Adhes. 1969, $1,102-123$.

25. Owens, D.K.; Wendt, R.C. Estimation of the surface free energy of polymers. J. Appl. Polym. Sci. 1969, 13, 1741-1747.

26. Scholze, H. Glas-Natur, Struktur und Eigenschaften, 3rd ed.; Springer Verlag: Berlin Heidelberg, Germany, 1988. (In German)

27. Carré, A. Polar interactions at liquid/polymer interfaces. J. Adhes. Sci. Technol. 2007, 21, 961-981.

28. Bach, H.; Krause, D. Analysis of the Composition and Structure of Glass and Glass Ceramics, 1st ed.; Springer Verlag: Berlin Heidelberg, Germany, 1999.

29. Gerhard, C.; Weihs, T.; Luca, A.; Wieneke, S.; Viöl, W. Polishing of optical media by dielectric barrier discharge inert gas plasma at atmospheric pressure. J. Eur. Opt. Soc. Rapid Publ. 2013, 8, doi:10.2971/jeos.2013.13081.

30. Lawrence, J.; Li, L. Wettability characteristics of an $\mathrm{Al}_{2} \mathrm{O}_{3} / \mathrm{SiO}_{2}$-based ceramic modified with $\mathrm{CO}_{2}$, Nd:YAG, excimer and high-power diode lasers. J. Phys. D 1999, 32, 1075-1082.

31. Tasche, D.; Gerhard, C.; Ihlemann, J.; Wieneke, S.; Viöl, W. The impact of O/Si ratio and hydrogen content on ArF excimer laser ablation of fused silica. J. Eur. Opt. Soc. Rapid Publ., in press.

32. Gerhard, C.; Roux, S.; Brückner, S.; Wieneke, S.; Viöl, W. Low-temperature atmospheric pressure argon plasma treatment and hybrid laser-plasma ablation of barite crown and heavy flint glass. Appl. Opt. 2012, 51, 3847-3852.

33. Gerhard, C.; Roux, S.; Brückner, S.; Wieneke, S.; Viöl, W. Atmospheric pressure argon plasma-assisted enhancement of laser ablation of aluminum. Appl. Phys. A 2012, 108, 107-112.

34. Gerhard, C.; Roux, S.; Peters, F.; Brückner, S.; Wieneke, S.; Viöl, W. Hybrid laser ablation of $\mathrm{Al}_{2} \mathrm{O}_{3}$ applying simulataneous argon plasma treatment at atmospheric pressure. J. Ceram. Sci. Technol. 2013, 4, 19-24.

(C) 2014 by the authors; licensee MDPI, Basel, Switzerland. This article is an open access article distributed under the terms and conditions of the Creative Commons Attribution license (http://creativecommons.org/licenses/by/3.0/). 